\title{
Design and Construction Method of an Open-Type Magnetically Shielded Room for MRI Composed of Magnetic Square Cylinders
}

\author{
Shigetaka Hirosato $^{1}$, Keita Yamazaki ${ }^{1}$, Yu Haraguchi ${ }^{2}$, Kazuhiro Muramatsu ${ }^{2}$, Akira Haga $^{3}$, Kiyotaka Kamata ${ }^{4}$ \\ Koichiro Kobayashi ${ }^{5}$, Atsuo Matsuura ${ }^{5}$, and Hitomi Sasaki ${ }^{5}$ \\ ${ }^{1}$ Research \& Development Institute, Takenaka Corporation, Chiba 270-1395, Japan \\ ${ }^{2}$ Department of Electrical and Electronic Engineering, Saga University, Saga 840-8502, Japan \\ ${ }^{3}$ Department of Electrical Engineering \& Information Technology, Tohoku Gakuin University, Miyagi 985-8537, Japan \\ ${ }^{4}$ Department of Electric Control Engineering, Kagoshima National College of Technology, Kagoshima 899-5193, Japan \\ ${ }^{5}$ Faculty of Engineering, Iwate University, Iwate 020-8551, Japan
}

\begin{abstract}
To shield the leakage flux densities of $3 \mathrm{~T}$ magnetic resonance imaging (MRI) higher than $1.5 \mathrm{~T}$ MRI and to reduce the degradation of magnetic properties during construction, the design of an open-type magnetically shielded room (MSR) combined with square cylinders made of magnetic and conductive materials should be improved. This paper reports on an optimal design for magnetic cylinders to improve the shielding effect, while also considering ease of construction with a view to practical use. Firstly, the structure of the magnetic cylinders, such as their opening width and the slit width were optimized using a 3D linear magnetic-field analysis. Using optimal magnetic cylinders, when the relative permeability $\left(\mu_{\mathrm{r}}\right)$ of the magnetic material is larger than 10000 , the reluctance of gaps between the cylinders is the main contributor to the leakage flux. So, the required $\mu_{\mathrm{r}}$ of the magnetic material is 10000 and oriented silicon steel is adopted instead of the non-oriented type. Secondly, as an optimal construction method, it was determined by experiment that oriented silicon steel sheets, annealed after bending, should be laminated so that the effect of gaps on butt joints between steel sheets on the magnetic property of the cylinder becomes smaller. This enables the relative permeability of 10000 when the maximum flux density of the material in the cylinder is less than $1.7 \mathrm{~T}$. Finally, the minimum thickness of oriented silicon steel sheet of cylinders depending on the distance from a 3 T MRI is obtained using linear magnetic analysis under the condition that the maximum flux density of the material of the cylinder is less than 1.7 T. In the case of the thickness of $3.8 \mathrm{~mm}$, the leakage flux density of $3 \mathrm{~T}$ MRI is decreased to less than a guideline $(0.5 \mathrm{mT})$ when the distance between the cylinders and the center of MRI is larger than $2750 \mathrm{~mm}$. This indicates that the optimal design and construction method of an open-type MSR for 3 T MRI composed of magnetic square cylinders are realized.
\end{abstract}

Index Terms_Leakage flux, magnetic field analysis, magnetically shielded room, magnetic resonance imaging.

\section{INTRODUCTION}

$\mathbf{I}$ N ORDER to reduce the leakage flux density to less than a guideline $(0.5 \mathrm{mT})$ [1] outside the room and shield an electro-magnetic field at high-frequency for operating, a magnetic resonance imaging (MRI) scanner is usually installed in a magnetically shielded room (MSR) surrounded by a wall with magnetic and conductive layers. The small space enclosed by the wall causes patients a great deal of stress and discomfort. In order to ameliorate this problem, several open-type MSRs for MRIs have been proposed, in which magnetic bars [2], canceling coils [3], or magnetic and conductive square cylinders [4] are used for shielding the magnetic field, and shielded glass is used for shielding the electro-magnetic field. Among these open-type MSRs, the cylinder type seems to be attractive because the number of panes of shielded glass, which are expensive and not sufficiently transparent, can be diminished by the wave-guide effect of the conductive cylinders, and the construction method of stacking the cylinders enables easy assembly and dismantling for moving and replacing the MRI device. In Yamazaki et al. [4], the concepts of this MSR, the basic optimal structure of the cylinders, and the possibility of its realization have been described.

Manuscript received March 06, 2009. Current version published September 18, 2009. Corresponding author: S. Hirosato (e-mail: hirosato.shigetaka@takenaka.co.jp).

Color versions of one or more of the figures in this paper are available online at http://ieeexplore.ieee.org.

Digital Object Identifier 10.1109/TMAG.2009.2025259
However, to shield higher flux densities of MRIs higher than 1.5 T MRI and reduce the degradation of the magnetic properties in construction, it is necessary to establish an improved design method taking account of ease of construction with a view to practical use. In particular, investigations of the structure of the magnetic cylinders are important because the flux densities of MRIs become higher and the magnetic characteristics of the cylinders are deteriorated by the gaps due to the presence of the conductive cylinders and distortions introduced during construction for bending and cutting of magnetic sheets in the cylinders. This paper describes an optimal design and construction method of magnetic cylinders for higher flux density MRIs and reducing the degradation of the magnetic properties.

In this paper, first, the structure of the magnetic cylinder taking account of ease of construction is optimized using a 3D linear magnetic-field analysis. By analyzing the relationship between the relative permeability $\left(\mu_{\mathrm{r}}\right)$ of magnetic cylinders and shielding effect is discussed to obtain the required $\mu_{\mathrm{r}}$. Second, a method of stacking magnetic sheets without degradation of the magnetic properties is discussed based on experiment. Finally, the thickness of cylinders required to realize the open-type MSRs designed in this paper is discussed.

\section{Optimization of MAGNETiC SQUARE CYLINDER}

\section{A. Analysis Model and a Method of Analysis}

The optimal design of a magnetic square cylinder is carried out using the model shown in Fig. 1. First, using a 3D linear magnetic-field analysis, the effects of the opening width, $W$, length, $L$, and thickness, $t$, of the cylinders on the shielding 


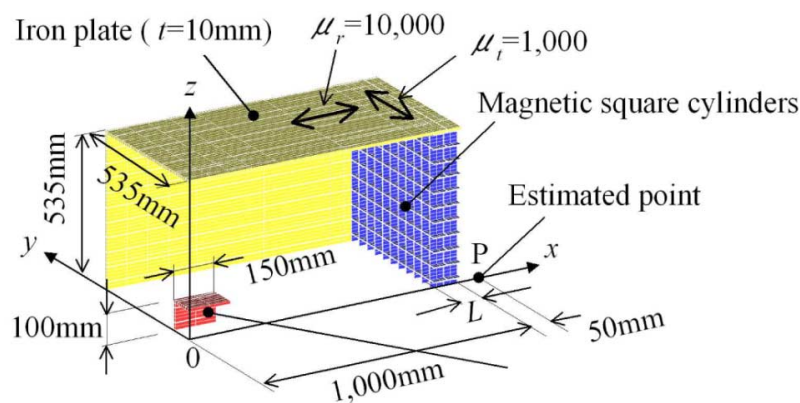

(a)

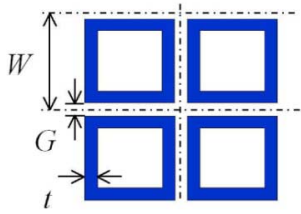

$y-z$ plane

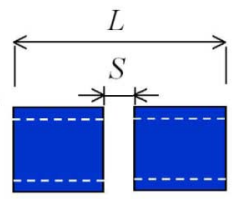

$x-z$ plane

(b) $\mu_{r}=10,000 \quad \mu_{t}=1,000$

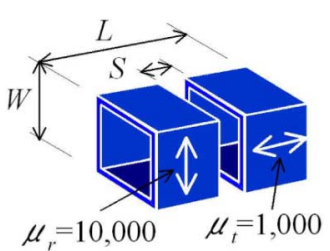

TABLE I

SIZE AND THE $S F$ OF THREE MODELS

\begin{tabular}{|c|c|c|c|c|c|c|}
\hline Model & $W$ & $L$ & $t$ & $G$ & $S$ & $S F$ \\
\hline A & 150 & 300 & & & & 17.8 \\
\hline B & \multirow{2}{*}{50} & 100 & & \multirow{3}{*}{5} & \multirow{2}{*}{3} & 3.47 \\
\cline { 1 - 1 } & & 50 & 2 & & & 2.75 \\
\hline
\end{tabular}

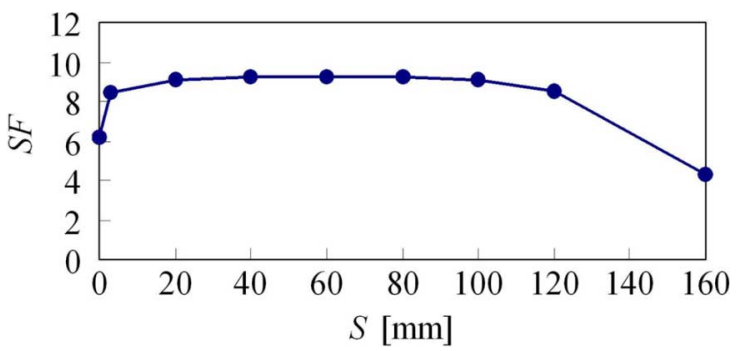

Fig. 3. Effect of the slit width $S$, on the $S F$. ( $W=150, L=300, t=1, G=$ $5)$

the total thickness of wall in the ordinary MSR. So, in the section below, the optimal design of cylinder are discussed under the condition of $L=300 \mathrm{~mm}$.

2) Examination of the Slit Width $S$ : In a previous report [4], the shielding effect of a magnetic cylinder divided into three sections in the direction of the depth $(x)$ was $3 \%$ higher than that divided into two sections. From the viewpoint of ease of construction, the number of divisions should be reduced. Therefore, the cylinder divided into two sections is employed. Fig. 3 shows the effect on the $S F$ when the slit width, $S$, is changed. A larger $S$ results in a larger total magnetic reluctance in the $x$-direction of the cylinders. Also, the $S F$ increases as the leakage flux to outside is reduced. In the case of $S$ larger than $100 \mathrm{~mm}$, the volume of magnetic material is smaller so the $S F$ decreases. In the range of optimal $S$, between 20 and $100 \mathrm{~mm}$, an $S$ of 80 $\mathrm{mm}$ is adopted.

In addition, expanding the slit width from $30 \mathrm{~mm}$ in [4] to 80 $\mathrm{mm}$ and diminishing the number of divisions from three to two contribute to easy construction and weight saving.

3) Examination of the Opening Width $W$ : In this section, the weight, wall thickness, and slit width are constant, and the optimal value of opening width $W$ is investigated. Fig. 4 shows the effect of the opening width $W$ on the $S F$. The thickness $t$ of the cylinder also changes by following $W$ under the condition mentioned above. As the opening width, $W$, is enlarged, the number of gaps, $G$, between the cylinders becomes fewer. As a result, the magnetic reluctance of the $y$ - and $z$-directions becomes small, and the $S F$ improves. However, when $W$ becomes larger than $250 \mathrm{~mm}$, the flux cannot be caught by cylinders. This results in increased leakage of the magnetic field to the outside and a decrease in the $S F$. The optimal $W$ of $210 \mathrm{~mm}$ is adopted in this model, and the thickness $t$ is set to $1.4 \mathrm{~mm}$.

In addition, expanding $W$ from $150 \mathrm{~mm}$ in [4] to $210 \mathrm{~mm}$ and diminishing the number of cylinders contribute to improving in shielding effect and ease of construction.

4) Magnetic Permeability of Square Cylinders: In this MSR, the shielding effect depends not only on the magnetic reluctance of the gap between the cylinders but also on that of the magnetic materials. So, the relation between the relative permeability and thinner $t$ enables a higher $S F$. However, the $L$ of the MSR for MRI should be restricted to less than about $300 \mathrm{~mm}$, which is bers of gaps. As a result, the $S F$ of Model A is higher than that of Models B and C.

As mentioned above, when the total weight of magnetic material is uniform, the combination of increased $W$ and $L$, and 


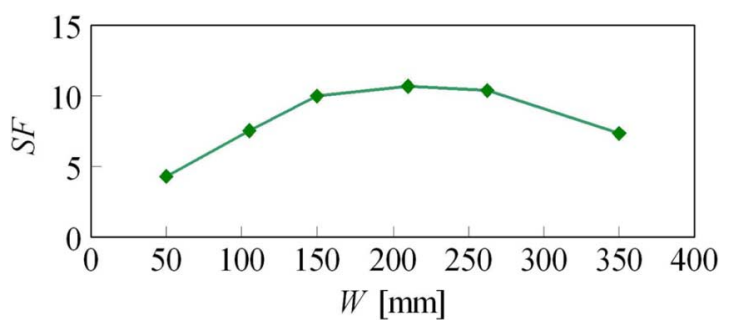

Fig. 4. Effect of the width, $W$, on the $S F,(L=300, S=80, G=5$ constant weight).

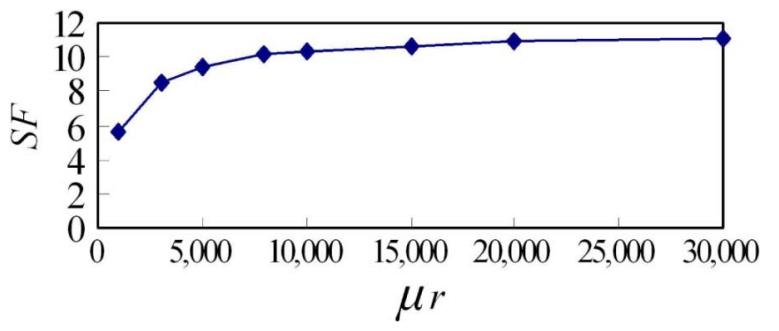

Fig. 5. Effect of the relative permeability on the $S F$. ( $W=210, L=300, t=$ $1.4, S=80, G=5$ ).

shielding effect is analyzed. Fig. 5 shows the $S F$ depending on the relative permeability $\mu_{\mathrm{r}}$ of magnetic materials.

In this figure, the $S F$ increases as the relative permeability, $\mu_{\mathrm{r}}$, increases below $\mu_{\mathrm{r}}=10000$. Then, as $\mu_{\mathrm{r}}$ increases above $\mu_{\mathrm{r}}=10000$, the $S F$ does not increase significantly. This indicates that above $\mu_{\mathrm{r}}=10000$, the magnetic reluctance of gap between cylinders is the main contributor to the leakage flux. This indicates that the required $\mu_{\mathrm{r}}$ of the magnetic material is 10000 . As a magnetic material, oriented silicon, whose $\mu_{\mathrm{r}}$ can be 10000 , is adapted instead of non-oriented silicon [4]. Even if the properties of the magnetic material are deteriorated by cutting and bending, an oriented silicon steel sheet is expected to retain the $\mu_{\mathrm{r}}$ of 10000 .

\section{Construction Method of Magnetic Cylinder}

In [5], using plain sheets, the $\mu_{\mathrm{r}}$ of 10000 is retained at $B$ less than $1.7 \mathrm{~T}$. The magnetic cylinder should retain the $\mu_{\mathrm{r}}$ of 10000 at $B$ less than $1.7 \mathrm{~T}$, as in [5]. However, when the cylinder is formed by combining sheets, the gaps occurs on the butt joint and the magnetic property of cylinder becomes worse. Therefore, we discuss a method for the construction of the magnetic cylinders based on experiments.

Fig. 6 shows several models. In Models D and E, each layer is divided into two parts. At the corners, the thickness of the sheets is diminished to half compared to the plain part. In Model $\mathrm{E}$, the sheets are annealed after bending. In Models F and G, each layer is divided into four parts and the joints of each layer are not located at the same position. In Model G, the sheets are annealed after bending. The magnetization coil shown in Fig. 7 is set to each model, and a current of $10 \mathrm{~Hz}$ is applied to excite the magnetic sheet. The magnetic field $H$ is calculated from the length of the magnetic material, the number of turns in the coil, and the current value. $B$ coil (10 turns) for calculating the flux density is simultaneously set to each model as shown in Fig. 7. The flux density, $B$, is then calculated from the waveform of

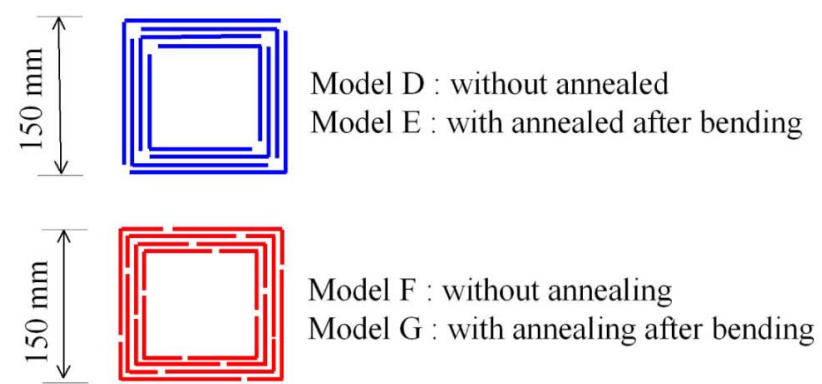

All Models are made from the oriented silicon steel, $t=0.35 \mathrm{~mm} \times 4$ Layer

Fig. 6. Composing methods for magnetic cylinders.

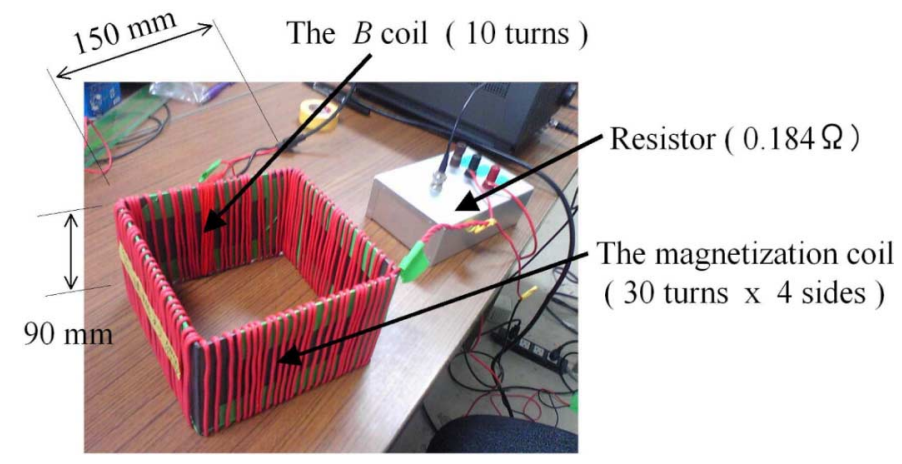

Fig. 7. Experimental model for measuring $\mu_{\mathrm{r}}-B$ curve.

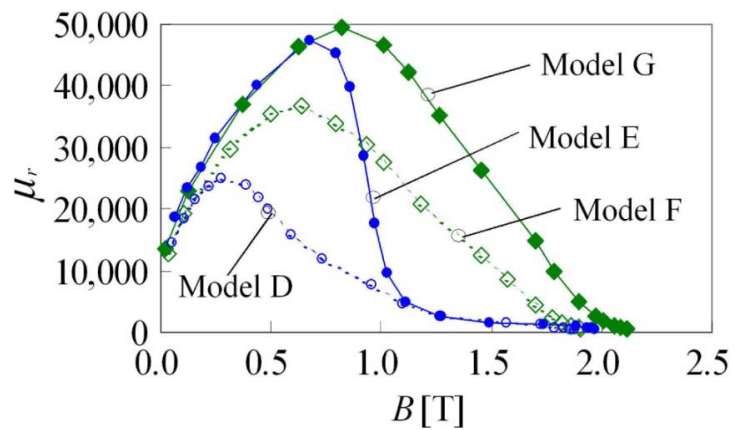

Fig. 8. $\mu_{\mathrm{r}}-B$ curve of each model.

the voltage of the $B$ coil. The magnetization current is changed from 0.2 to $20 \mathrm{~A}$.

Fig. 8 shows the measured $\mu_{\mathrm{r}}-B$ curve of each model. It is suggested that Models D and E are magnetically saturated because the thickness of the sheets at the corners are diminished to half compared to the plain parts. In Model G, degradation due to bending at the corners is improved by annealing. So, the magnetizing properties of Model $\mathrm{G}$ are better than Model F.

We adopt the following construction method for the magnetic cylinders: each layer is divided into four parts and the joints of each layer are not located at the same position. The sheets are annealed after bending.

When the flux density $B$ is less than $1.7 \mathrm{~T}$, it is verified that the relative permeability becomes 10000 .

\section{The Analysis Method IN the Actual MRI}

We compute the minimum thickness of magnetic cylinder in linear analysis, depending on the distance from an actual $3 \mathrm{~T}$ 


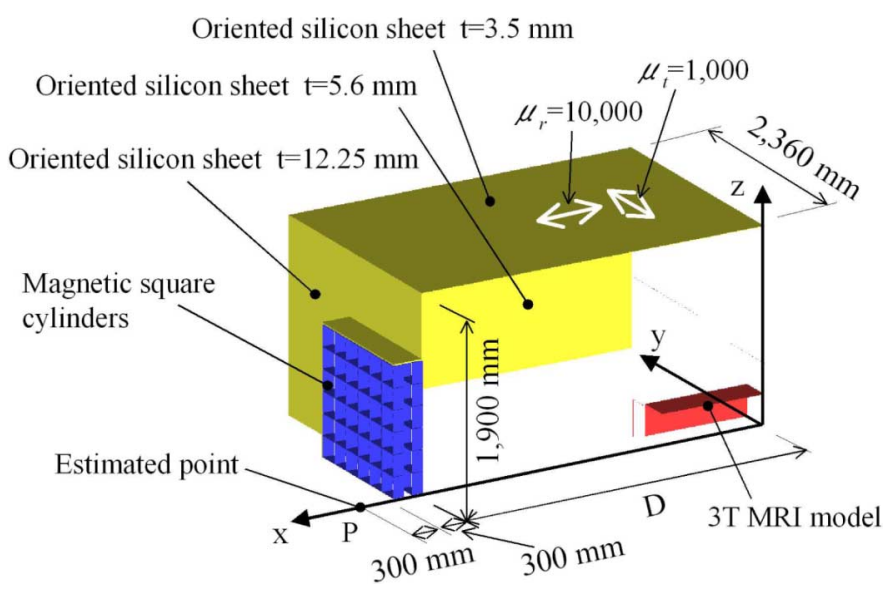

Fig. 9. Analyzed model of actual MSR and 3 T MRI model. (1/8 region model).

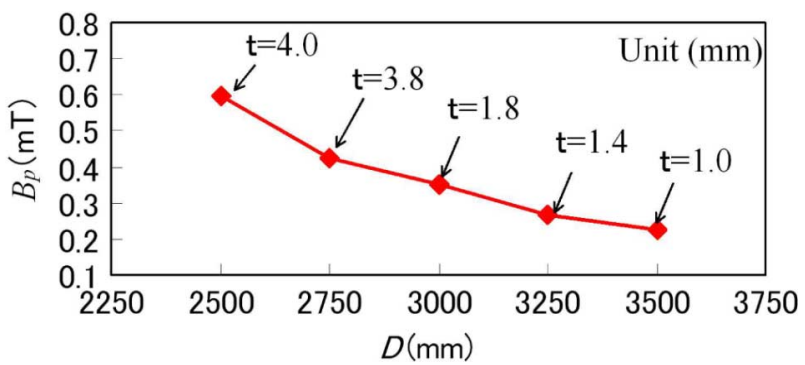

Fig. 10. Leakage magnetic field at point, p, of depending on the distance between the cylinders and the center of MRI and thickness, $t$, of magnetic cylinders, under the condition that the maximum flux density $B$ of cylinder is less than $1.7 \mathrm{~T}$.

MRI, under the condition that the maximum flux density $B$ of cylinder is less than $1.7 \mathrm{~T}$, as described above. This results in the $\mu_{\mathrm{r}}$ of the magnetic cylinder being 10000 for oriented silicon steel. Fig. 9 shows the analyzed of the actual MSR for 3 T MRI. However, only $1 / 8$ region model is employed since the fields are symmetrical. Width, length and height of this MSR are 4 $720 \mathrm{~mm}, 6000 \mathrm{~mm}$ and $3800 \mathrm{~mm}$, respectively. Opening of the wall for stacking the cylinders is $2520 \mathrm{~mm}$ of width and $2520 \mathrm{~mm}$ height. The current value of a coil is determined to accord with the leakage magnetic field of 3 T MRI [6]. The 144 pieces of the cylinders with $W=210, L=300$, and $S=80$ $\mathrm{mm}$ determined according to the optimal design in the previous section are stacked. The number of elements is 461563 . The relative permeabilities are shown in Fig. 9.

Fig. 10 shows the leakage flux density, $B_{\mathrm{p}}$, at the point, $\mathrm{p}, 300 \mathrm{~mm}$ outside from cylinders depending on the distance $D$, between the cylinders and the center of MRI. $B_{\mathrm{p}}$ is decreased to less than a guideline $(0.5 \mathrm{mT})$ when $D$ is larger than $2750 \mathrm{~mm}$. The minimum thicknesses of the cylinders for $D=2750,3000,3250$ and $3500 \mathrm{~mm}$ are $3.8,1.8,1.4$, $1.0 \mathrm{~mm}$, respectively. Therefore, it is demonstrated that the optimal design and the construction method of an open-type MSR composed of magnetic square cylinders are realized for 3 $\mathrm{T}$ MRI when the distance between the cylinders and the center of MRI is larger than $2750 \mathrm{~mm}$.

\section{CONCLUSION}

For the purpose of improving the shielding effect and construction, the optimal design of cylinders is investigated by analysis and experiment. The obtained results are summarized as follows.

1) The opening width of $150 \mathrm{~mm}$, with 2 slits, and a slit width of $30 \mathrm{~mm}$ in previous designs were improved to $210 \mathrm{~mm}$, 1 slit, and $80 \mathrm{~mm}$. These result in improvements of the shielding effect and ease of construction.

2) In this MSR, for magnetic materials with $\mu_{\mathrm{r}}$ above 10000 , the magnetic reluctance of gap between cylinders is the main contributor to the leakage flux. So, the required $\mu_{\mathrm{r}}$ of the magnetic materials is 10000 and the oriented silicon steel sheet is adopted instead of the non-oriented one used in previous design.

3) As the magnetic material of cylinders, oriented silicon steel sheets annealed after bending should be used. These should be laminated so that the positions of gaps on butt joints between sheets are different each other.

4) The total thickness of oriented silicon steel sheets in the cylinders obtained using linear magnetic analysis under condition that the maximum flux density of the material of the cylinder is less than $1.7 \mathrm{~T}$ can decrease leakage magnetic field of $3 \mathrm{~T}$ MRI to less than a guideline $(0.5 \mathrm{mT})$ when the distance between the cylinders and the center of MRI is larger than $2750 \mathrm{~mm}$. This indicates that the optimal design and construction method of an open-type MSR composed of magnetic square cylinders are realized for 3 T MRI.

Next, the validity of optimal design of cylinders for 3 T MRI is verified by the nonlinear analysis and measurement of distribution of magnetic flux of 3 T MRI installed in the hospital.

\section{REFERENCES}

[1] "Guideline to limits of exposure to static magnetic fields," Health Phys., vol. 66, pp. 113-122, 1994, ICNIRP.

[2] T. Saito, IEEE Trans. Magn., vol. 44, no. 11, p. 4191, Nov. 2008.

[3] S. Hirosato et al., IEEE Trans. Magn., vol. 42, no. 10, p. 3542, Oct. 2006.

[4] K. Yamazaki et al., IEEE Trans. Magn., vol. 44, no. 11, p. 4183, Nov. 2008.

[5] "Direct-current magnetization curve," Direct-Current Amplitude Permeability Curve 1988, Nippon Steel Corporation, Cat. No. DE105 P154 "35Z155".

[6] Signa HDx 3.0 T Pre-Installation Copyright c 2006 by General Electric Company Inc.. 